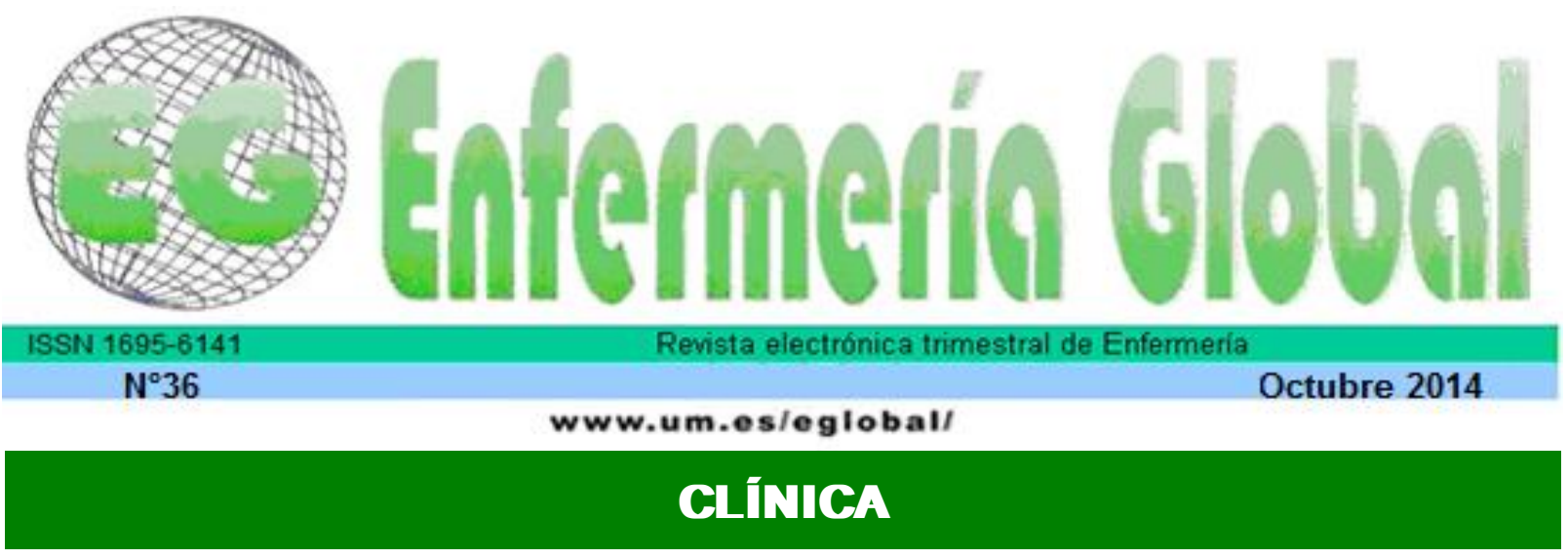

\title{
Enfermera Gestora de Casos del Servicio Murciano de Salud: Un año de puesta en marcha del programa
}

Nurse Case Manager Murcia Health Service: A year of program implementation

\begin{abstract}
*Valverde Jiménez, María del Rosario **López Benavente, Yolanda *Hernández Corominas, María Ángeles ${ }^{* * *}$ Gómez Ruiz, Mercedes ****Bázquez Pedrero, Magina *****Echevarría Pérez, Paloma

\author{
*Enfermera Gestora de Casos del Servicio Murciano de Salud. E-mail: mariar.valverde@carm.es \\ **Enfermera Servicio Murciano de Salud ${ }^{* * *}$ Coordinadora Regional de Enfermería del Servicio \\ Murciano de Salud ${ }^{* * * *}$ Directora General de Asistencia Sanitaria del Servicio Murciano de Salud \\ *****Decana de la Facultad de Enfermería de la Universidad Católica de San Antonio (UCAM). Murcia.
}

Palabras clave: gestor de salud; investigación; atención de enfermería

Keywords: health manager; research; nursing care

\section{RESUMEN}

Los objetivos que se plantean son describir los procesos claves de la enfermera gestora de casos (EGC) en un año de puesta en marcha del programa. Conocer el perfil sociodemográfico y clínico de la población diana y conocer la sobrecarga en el cuidador familiar.

Método; El estudio es observacional descriptivo y transversal en el Área IX de salud Murcia desde el 1 de junio de 2010 hasta 31 mayo de 2011. Se realiza mapa de procesos de la EGC, identificación de pacientes con OMI-AP y CIVITAS, y se realiza visita en consulta y/o domiciliaria para valoración inicial con datos sociodemográficos, 11 patrones funcionales de M. Gordon, y cuestionarios validados. El número de personas captadas es de 258 pacientes y 115 cuidadoras. La población con EPOC representa un 22,9\%. El 63,9\% de pacientes tienen valoración completa realizada.

Resultados: El patrón actividad y ejercicio es el más alterado. Los pacientes tienen una media de edad de $74 \pm 15$ años. Las mujeres predominan en la población diana general $54,7 \%$. El $87,2 \%$ presenta algún grado de dependencia, existe un mayor porcentaje con dependencia severa. La media de edad de la cuidadora familiar, es $59 \pm 13$ años, de las cuales, el $40 \%$ de las cuidadoras familiares no tienen sobrecarga. La población captada supone un $5,3 \%$ de la población compleja. Desde la incorporación de la EGC, ha aumentado el registro de las valoraciones por patrones, tanto de pacientes como de cuidadoras. La población captada está envejecida, frágil, con un nivel de dependencia severo, preferentemente es mujer, con cuidadora familiar (mujer) donde son más frecuentes las que no tienen sobrecarga. 


\title{
ABSTRACT
}

The objectives of this paper are to describe the fundamental processes of the nurse case manager in a year of the program, to know the sociodemographic and clinical profile of the target population and the strain on the caregiver.

\begin{abstract}
Methodology: This observational, descriptive and transversal study has been made in the health area of Murcia IX from 1 June 2010 to 31 May 2011. It describes the process maps of the nurse case manager; patients are identified through OMI-AP software and CIVITAS. Home visits are conducted to measure the social and demographic patterns, to perform evaluation through M. Gordon functional patterns and to apply validated questionnaires. The number of people who enter into the program is 258 patients and 115 caregivers. The population with chronic obstructive pulmonary disease represents $22.9 \% ; 63.9 \%$ of patients have made full assessment.
\end{abstract}

Results: The functional pattern that measures activity and exercise is the most altered. Patients have a mean age of $74 \pm 15$ years. Women predominate in the overall target population $54.7 \% ; 87.2 \%$ have some degree of dependence, there are a higher percentage of patients with severe dependence. The average age of caregivers is $59 \pm 13$ years, of which, $40 \%$ of family caregivers are not overloaded. The target population represented $5.3 \%$ of the population more vulnerable. Since the introduction of case manager, registration of functional patterns in medical records of patients and caregivers has increased. The aging population is high, fragile, with a severe dependence level, preferably female, with family caregivers (women) without overloading conclusions

\section{INTRODUCCIÓN}

El conocimiento de las necesidades de la población en general, de cada uno de los sectores en particular, la redistribución de los recursos tanto a nivel sanitario como social, son factores que influyen para saber el nivel de bienestar social. Es importante tener en cuenta que para cubrir las necesidades socio-sanitarias de la población es necesario un funcionamiento coordinado de los sistemas de protección de la salud y de los servicios sociales ya que no siendo así, se produce un derroche de recursos y la existencia de carencias para la población por no estar cubiertas sus necesidades. Estas situaciones son detectadas habitualmente por los servicios sanitarios ${ }^{1}$. La gestión de casos se presenta como un enfoque al desarrollo de una atención integral sociosanitaria.

La gestión de casos aparece por primera vez en Estados Unidos, se puso en marcha ante la falta de un sistema integral de cobertura y la fragmentación de agencias existentes ${ }^{2}$. La gestión de casos resurge en el sistema de atención a la salud a finales de los 60 y comienzos de los 70. Aparece como elemento fundamental del movimiento de desinstitucionalización ${ }^{3}$, orientado para la coordinación de servicios en personas con requerimientos de acciones más complejas en la comunidad. Después del éxito, tras las evaluaciones de los distintos programas en Estados Unidos, la gestión de casos se ha ido extendiendo a otros países, especialmente Gran Bretaña, Canadá, Australia, Israel, Holanda...Este éxito se debe a su habilidad para promover la coordinación sin implicar grandes cambios estructurales en los servicios sociosanitarios ${ }^{4}$.

El Case Management Society of America ${ }^{4}$ la define como "un proceso de colaboración mediante el que se valora, planifica, facilita, coordina, monitoriza y evalúa las opciones y servicios necesarios para satisfacer las necesidades de salud integrales de una persona y su familia, articulando la comunicación y recursos disponibles para promover resultados de calidad y costo-efectivos". 
Diversos autores explican que la gestión de casos no es una profesión sino que se trata de un método ${ }^{5}$ que requiere del desarrollo de unas competencias que incluyen capacidades para trabajar con problemas complejos, con las familias y sus múltiples necesidades, con numerosos interlocutores públicos, privados y empresariales y de lidiar con el sistema burocrático. Otros autores recomiendan que para la gestión de casos, se requiera de una especialización de posgrado ${ }^{6}$ que permita adquirir conocimientos sobre modelos, métodos, técnicas de planificación, evaluación y gestión de la calidad ${ }^{1}$ así como habilidades específicas en observación y comunicación.

Las personas que son susceptibles de gestión de casos son personas frágiles, vulnerables o complejas con una discapacidad funcional, escasez de recursos sociofamiliares, aislamiento social, que necesitan apoyo en las actividades básicas, así como en las actividades instrumentales de la vida diaria, que necesitan ayuda en el manejo de los síntomas, en el régimen terapéutico, que demandan múltiples recursos de la red asistencial y que tienen una necesidad de cuidados multidisciplinares y continuados $^{7}$

Según Kaiser Permanente ${ }^{8}$ (organización americana, sin ánimo de lucro, orientada al cuidado de la salud), ubicaría a los pacientes complejos entre el 3 y el $5 \%$ de la población (figura 1), en los que se encontrarían los pacientes pluripatológicos.

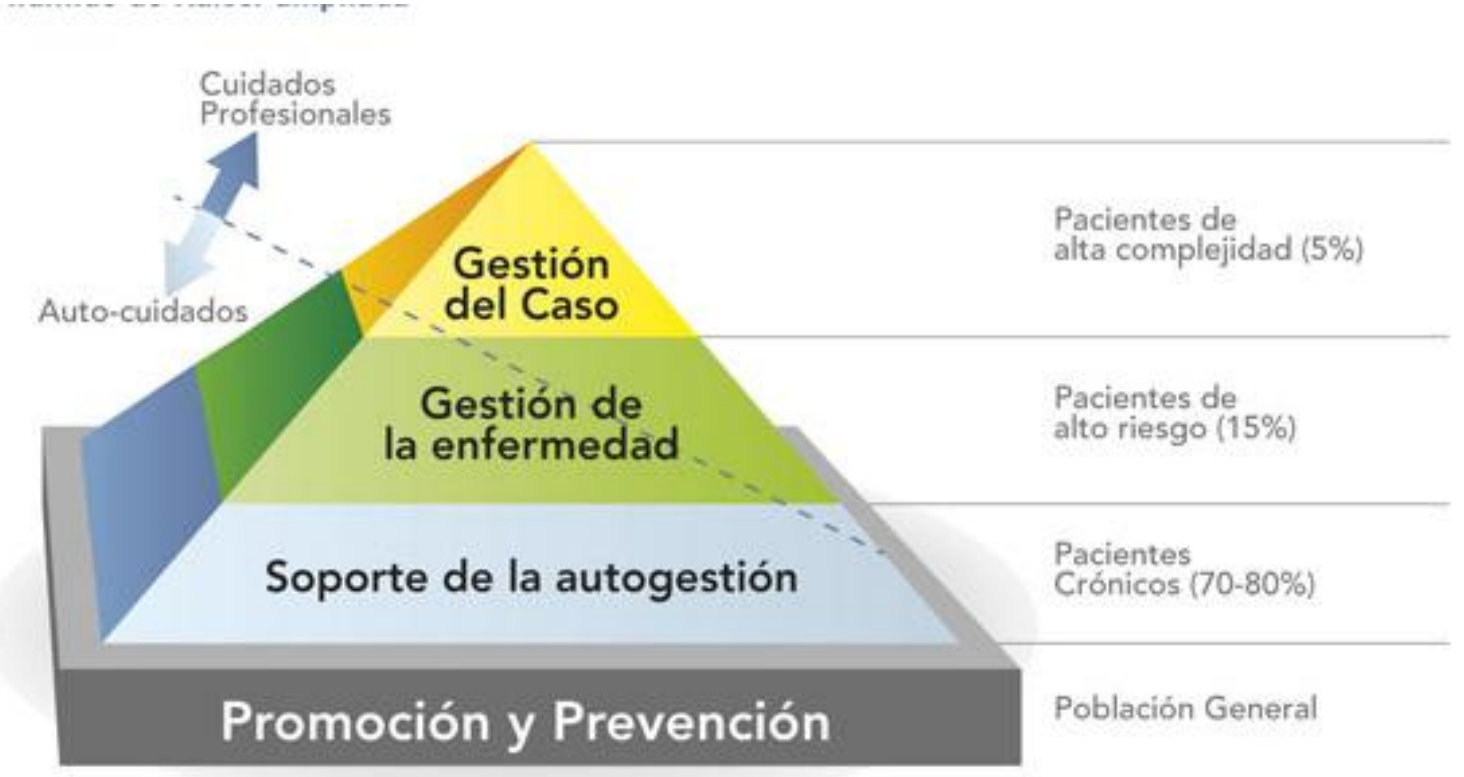

Figura 1 Fuente: Manuel Ollero. 2009:2. Gestión de población: modelo pirámide de riesgo

Las enfermeras son líderes en la disciplina del cuidado de la salud ${ }^{9}$ donde se centran en la respuesta de las personas derivadas de su ciclo vital y no sobre la enfermedad en sí. En la literatura, se identifican varias denominaciones para la enfermera gestora de casos como es la enfermera comunitaria de enlace, enfermera de continuidad de cuidados así como enfermera de gestión domiciliaria, donde el objetivo principal es el coordinar la continuidad del cuidado.

En líneas generales, no hay una política común u homogénea en cuanto a la implantación de la enfermera gestora de casos en las diferentes Comunidades Autónomas. Existen EGC que solamente se encuentran en atención primaria, otras 
desarrollan su trabajo en el hospital y no en la comunidad, o como es el caso de Andalucía, donde se desarrolla el trabajo de gestión de casos en hospital y domicilio pero con figuras de enfermeras de enlace diferentes.

El Plan de Mejora y Modernización de la Atención Primaria 2007-2010 ${ }^{10}$.Tras las transferencias, el SMS ha iniciado actuaciones diversas en relación a la continuidad asistencial en la que se destacan los apartados en relación con el trabajo de la EGC: "Atención primaria como principal gestor de necesidades y casos y la Atención domiciliaria y fuera del centro donde destaca la figura de enfermera comunitaria de enlace, asumiendo el papel de gestión de casos".

\section{Gestión por procesos en la Gestión de Casos de la Región de Murcia}

Dentro del campo profesional sobre gestión de casos, hay que definir cuál es nuestra área de responsabilidad autónoma y cómo se insertan de forma eficiente nuestras actividades. Se trata de trabajar bajo una visión estratégica ${ }^{11}$ que no esté basada en la improvisación y la incertidumbre (figura 2).

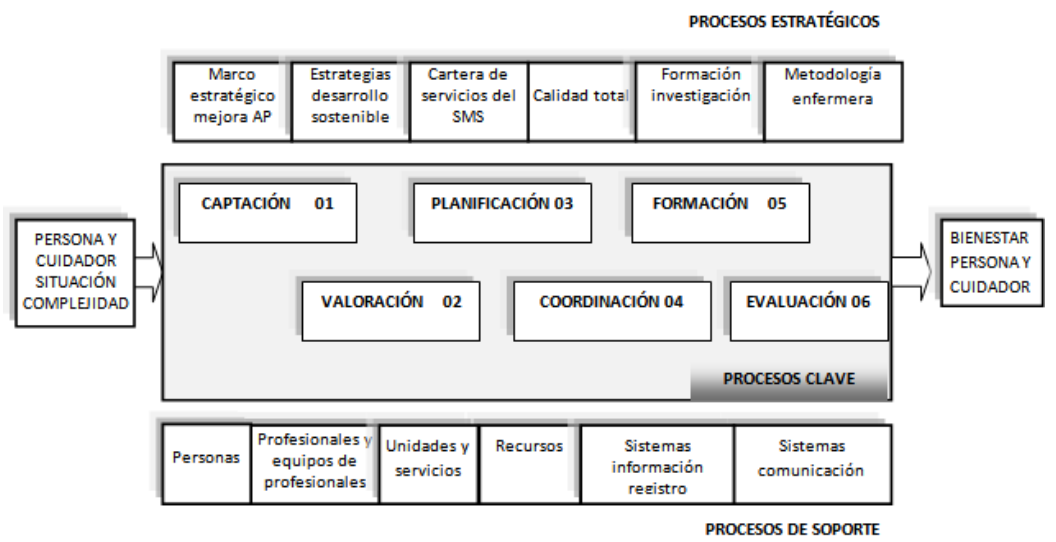

Figura 2. Fuente: Dirección General de Asistencia Sanitaria. 2010:7. Mapa de procesos

La gestión por procesos se organiza de forma estructurada y considera la visión del cliente como prioritaria. Sobre su mejora se basa la de la propia organización ${ }^{12}$. Es un elemento clave en un modelo de atención integrada y una herramienta de calidad para optimizar resultados, de adaptación a las necesidades de los clientes y de mejora de la satisfacción de los profesionales.

Se definen claramente componentes esenciales de la atención (figura 2): Captación, valoración, planificación, coordinación, formación y evaluación.

La creación de las Gerencias Únicas en el Servicio Murciano de Salud, unificando las gerencias de Atención Primaria con las gerencias de Atención Hospitalaria, supuso ofrecer una atención integral de las necesidades de salud de los ciudadanos, considerando la atención como un proceso longitudinal.

Para asegurar esta continuidad de cuidados, el ámbito de actuación de la enfermera gestora de casos en el SMS es toda el Área de Salud ${ }^{13}$, a diferencia de cómo hemos visto anteriormente, en otras Comunidades Autónomas se distingue la EGC domiciliaria y la EGC hospitalaria. 
El modelo de gestión de casos se implantó el 1 de junio de 2010, como proyecto piloto, con dos enfermeras gestoras de casos. Una EGC en la zona básica de salud de Cieza y otra EGC en las zonas básicas de salud de Abarán y Blanca. Su ubicación física es el centro de salud.

El interés de nuestro estudio es aportar una visión cercana del trabajo de la Enfermera Gestora de Casos en la Región de Murcia y la población diana a la que atiende, para priorizar las acciones de detección de necesidades biopsicosociales y así dirigir una planificación de cuidados garantes de calidad.

Los objetivos que se plantean en el trabajo son: describir la situación actual de los procesos clave del mapa de procesos de la enfermera gestora de casos en el Servicio Murciano de Salud en un año de puesta en marcha del programa, Conocer el perfil sociodemográfico y clínico de la población diana captada por la Enfermera Gestora de casos (EGC) y Conocer la sobrecarga en el cuidador familiar de la población diana.

\section{MATERIAL Y MÉTODO}

Para dar respuesta a los objetivos propuestos en nuestro estudio, se ha diseñado un estudio observacional descriptivo y transversal. El ámbito de estudio fue el Área IX de salud, Vega Alta del Segura (Murcia). El periodo de estudio fue desde el 1 de junio de 2010 hasta 31 mayo de 2011.

La Población de estudio está definida por las siguientes características: Personas afectadas por los siguientes procesos asistenciales, priorizando: Demencias: especialmente Alzeimer. Inmovilizados domiciliarios y especialmente aquellos con cambios frecuentes de residencia. Problemas de Salud Mental: especialmente Trastorno Mental Grave y adicción de alcohol y/o drogas. Personas dependientes. Enfermedades cardiovasculares en situación de prevención terciaria. VIH/SIDA. Hospitalizados y en domicilio con una situación de enfermedad crónica avanzada con descompensaciones: Insuficiencia cardiaca, insuficiencia renal, EPOC. Enfermedad en situación de terminalidad 0 de necesidad de Cuidados Paliativos. Personas hospitalizadas o en domicilio sin cuidados familiares o apoyo social. Personas en situación de alta hospitalaria con necesidad de cuidados domiciliarios continuados. Cuidador/a familiar de los anteriores.

Grupos de indicadores de inclusión: Grupo A: Deficiente red apoyo social: Escala de Valoración de riesgo social sociofamiliar de Gijón $>10$. Analfabetismo de la persona y/o cuidador. Claudicación familiar. Grupo B: Déficit de adherencia al tratamiento y/o seguimiento: no recogida de recetas de crónicos, y/o no acude a consulta de Atención Primaria en 2-3 ocasiones y/o no acude a consulta de Salud Mental en 10 más ocasiones. 3 ingresos hospitalarios en los últimos 6 meses por motivo de descompensación de su propia patología crónica. Grupo C: Oxigenoterapia domiciliaria. Mayores de 65 años y Polimedicados $>5$ fármacos y Situación de Riesgo de Dependencia o Fragilidad: Cuestionario de Barber $>1$.

Criterios de Inclusión: Presencia de 1indicador del Grupo A; Presencia de 2 indicadores del Grupo B; Presencia de 3 indicadores del Grupo C; Presencia de 1 indicador del B y 2 indicadores del C. El tamaño de la muestra incluyó a toda la población diana derivada a la enfermera gestora de casos, desde Atención Primaria, Atención Hospitalaria, Salud Mental, Comunidad y captación activa y que cumplieran criterios de inclusión. La muestra fue de 258 sujetos. 
La clasificación de variables se realiza en base a los procesos clave y a las características sociodemográficas y capacidad funcional del paciente. La evaluación de las variables del estudio se realizó a través de los protocolos de gestión de casos y planes de cuidados de la historia clínica electrónica de OMI-AP, a través del registro en hoja de recogida de datos ad hoc de la enfermera gestora de casos, y cuestionarios validados como el test de Barthel, cuestionario de Zarit y escala de valoración sociofamiliar de Gijón. La mayor parte de la recogida de los datos se llevó a cabo por las dos enfermeras gestoras de casos. También existen datos recogidos en OMI por los profesionales sanitarios que trabajan habitualmente con la población diana.

Se realizó un análisis descriptivo univariable ${ }^{14}$ de las variables cuantitativas, expresadas como media \pm desviación estándar, y las cualitativas, como número y porcentaje. Para el análisis estadístico comparativo bivariable entre variables cuantitativas y cualitativas de 2 categorías, se ha utilizado la t de Student, y la prueba de la ANOVA de un factor para cualitativas de más de 2 categorías. Tablas de contingencia para 2 variables cualitativas. La relación entre variables cuantitativas se ha realizado mediante el cálculo del coeficiente de correlación de Pearson. Se ha considerado estadísticamente significativo el valor de $p<0,05$. El análisis estadístico se ha realizado con el programa informático SPSS 15.0 para Windows.

\section{RESULTADOS}

Con fecha de explotación de datos de OMI del 15 de junio de 2010 y CIVITAS en marzo de 2010 (para personas mayores de > 14 años), se obtiene que el grupo de población diana con $\mathrm{n}^{\circ}$ de NIF con CIAP susceptibles de estar en la pirámide de riesgo (figura 2) es de 4833 personas en el Área ${ }^{15}$. El número de personas captadas en GC es de 258 (figura 3) es decir, un 5.3\% de la población susceptible de GC. Población total del área IX es de 54561 habitantes.

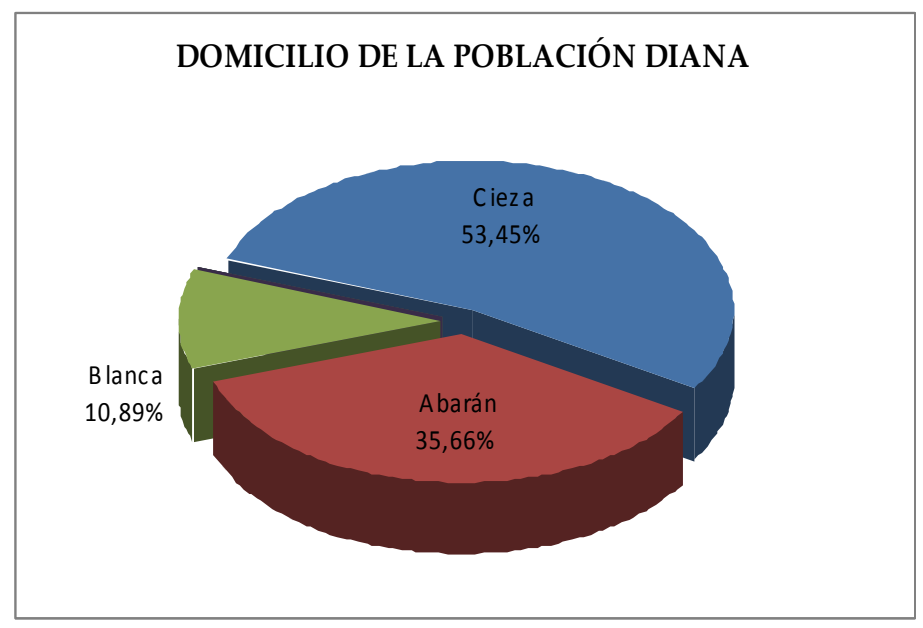

Figura 3. Fuente: elaboración propia. Distribución de la población por ZBS

La población con EPOC representa un 22,9\% (59), ACV 20,9\% (54); personas inmovilizadas y dependientes ocupan un 22,5\% (58), seguida del Trastorno Mental Grave (TMG) con un 10,1\% (26); Pacientes con demencia, especialmente tipo Alzeimer, ocupan un 9,3\% (24); personas sin apoyo social ocupan un 5,8\% (15); pacientes terminales un $5 \%$ (13) y pacientes con enfermedad cardiovascular en situación de prevención terciaria representan un 3,5\% (9). El grupo de población 
susceptible de gestión de casos "cuidadores familiares", captado por la EGC, es de 115 personas.

El 63\% (163) de los pacientes han sido captados por derivación desde A. P; el 20,9\% (54) desde Hospital; el 12,8\% (33) desde la Comunidad; el 3,1\% (8) por captación activa y no hay derivaciones desde Salud Mental (figura 13)

Dentro de los criterios de inclusión se obtiene que un 44,2\% (114) entran en programa por cumplir 3 criterios del grupo C; el 36,8\%(95) por una puntuación en la escala de valoración de riesgo socio-familiar de Gijón mayor de 10; el 14\% (36) por claudicación familiar; el 1,6\% (4) por 1 criterio del B y 2 del C; y el 1,2\% (3) por cumplir 2 criterios del B.

Al relacionar el grupo de población con el ámbito de derivación se observa que la población con ACV y EPOC es derivada desde AP con un 79,6\% y 83,1\% respectivamente y la población "no apoyo social" que es derivada desde atención hospitalaria en un $60 \%$. La población con TMG es derivada desde la comunidad representando un $57,7 \%$.

Cuando se relaciona la población diana con los criterios de inclusión se obtiene que la población con no apoyo social y TMG, el criterio de inclusión es deficiente red apoyo social: Escala de Valoración de riesgo socio-familiar de Gijón >10; y para la población con EPOC y ACV los criterios de inclusión son oxigenoterapia domiciliaria, anciano frágil, polimedicado y mayor de 65 años.

El no de pacientes con valoración completa realizada por patrones funcionales de $\mathrm{M}$. Gordon es del 63,9\%(129) frente al 36,1\% (73) que no se le ha realizado valoración completa, antes de la llegada de la enfermera gestora de casos, un 7,9\%(16) tuvieron registrada una valoración completa de los patrones funcionales, frente a un 92,1\% (186) que no se les había realizado valoración completa.

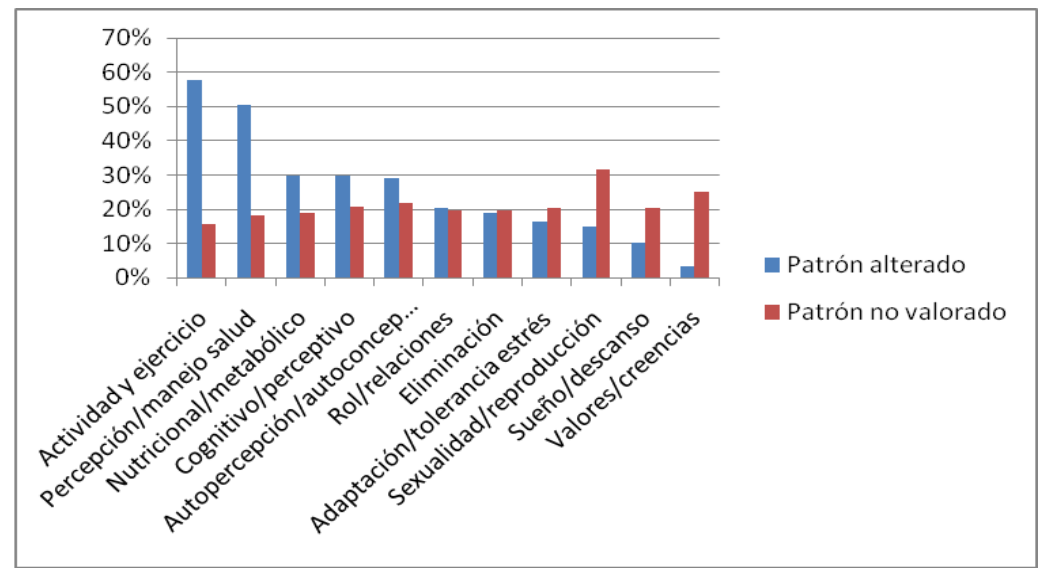

Figura 4. Fuente: elaboración propia. Gráfico de barras representativo de patrones alterados y no valorados en la población general.

Con respecto a los cuidadores, se han realizado valoración completa por la EGC, a un $16,7 \%$ (19), frente a los 0,9\% (1) que fueron valorados antes de la GC. Los resultados de la valoración de los 11 patrones funcionales muestran que el patrón actividad y ejercicio está alterado en un porcentaje mayor en la población representando el $58 \%$ 
(117), seguido del patrón percepción-manejo de la salud que representa el 50,5\% (102) (figura 4).

Las cuidadoras familiares presentan como patrón principalmente alterado el de actividad y ejercicio con un $65,76 \%$ seguido del patrón percepción manejo de la salud con un $58 \%$ (figura 5 ).

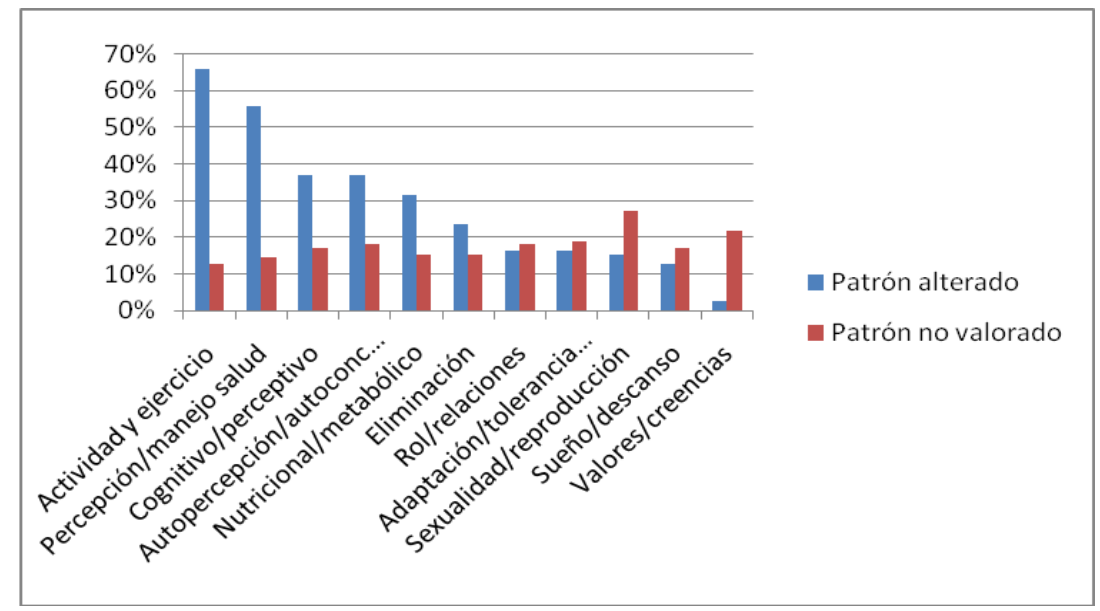

Figura 5. Fuente: elaboración propia. Gráfico de barras representativo de los patrones alterados y no valorados de las cuidadoras familiares

Los pacientes pertenecientes a la población diana Personas hospitalizadas o en domicilio sin cuidados familiares o apoyo social son los que presentan una frecuencia menor de valoración completa por patrones seguido de los pacientes con TMG.

Los patrones funcionales menos valorados en el total de la población a estudio son en primer lugar el patrón sexualidad-reproducción con un 31,68\% (64) seguido del patrón valores y creencias que representa el 25,2\% (51) (figura 14).

El porcentaje de pacientes con planes de cuidados completos realizados ha sido del $63,4 \%$ (128), frente a los 7,4\% (15) de planes realizados antes de que estuviera la EGC. Con respecto a las cuidadoras familiares, se le han realizado planes de cuidados completos a un $14,8 \%$ (17) mientras que, anteriormente a la GC, no se les habían realizado ningún plan de cuidados.

De los 258 casos derivados a la EGC, 183 casos siguen activos en GC. El 15,9\% (41) han salido de GC por éxitus, el 7,4\% (19) por seguimiento controlado, el 3,1\% (8) ingreso en residencia y el 2,7\% (7) han abandonado el programa.

Los pacientes que están en gestión de casos tienen una media de edad de $74 \pm 15$ años. Destacando que la población más envejecida está representada por los inmovilizados/dependientes, que tienen $80 \pm 10$ años, mientras que las personas con menor edad está representada por la población con TMG, que tiene una edad media de $44 \pm 15$ años.

En cuanto al sexo, las mujeres predominan en la población diana general con un $54,7 \%$ (141) y por grupos de población, las mujeres son más frecuentes en la población inmovilizada/dependiente con un 31,2\% (44), seguida de personas con ACV $23,4 \%$ (33) y demencia con $11,3 \%$ (16). Los hombres son más frecuentes en EPOC y TMG con un $35,9 \%$ (42) y $13,7 \%$ (16) respectivamente. 
Según el estado civil, la población casada y viuda tiene el mismo porcentaje 40,7\%. Por grupos de población, la población inmovilizadas/dependientes, ACV, demencia y no apoyo social hay en un mayor porcentaje viudos con un $26,7 \%$ (28), 22,9\% (24), $14,3 \%$ (15) y $6,7 \%$ (7) respectivamente. Los casados son más frecuentes en pacientes con EPOC 34,3\% (36), paciente terminal y pacientes con enfermedad cardiovascular en situación de prevención terciaria, ambos con 5,7\% (6). En los pacientes con TMG hay un mayor porcentaje de solteros $46,7 \%$ (21).

En cuanto a la relación entre grupo de población y cuidador se observa que las personas con TMG y personas hospitalizadas o en domicilio sin cuidados familiares 0 apoyo social, no tienen cuidador en un $65 \%$ y $80 \%$ respectivamente. Así como personas con ACV, demencia y EPOC sus cuidadores principales son familiares.

En la población estudiada el porcentaje de sujetos que presenta algún grado de dependencia para las actividades de la vida diaria, representa el 87,2\% (184), en concreto existe un mayor porcentaje de población con dependencia severa (Barthel 21-60) 30,8\% (65), seguida de dependencia moderada (Barthel 61-90) 27\% (57), dependencia total (Barthel 0-20) con un 25,1\% (53) y dependencia leve (Barthel 9199) que representa un 4,3\%(9). La población independiente (Barthel 100) representa un $12,8 \%(17)$.

Por grupo de población, las personas con demencia, presentan una dependencia total con mayor frecuencia 52,9\% seguida del ACV con un 51,9\%; los pacientes que tienen dependencia severa son los pacientes terminales representando un $75 \%$, los inmovilizados un $52,2 \%$ y los que no tienen apoyo social, representan el $37,5 \%$, los pacientes con EPOC presentan una dependencia moderada con un $43,6 \%$. Los más independientes para las ABVD son las personas con TMG con un $75 \%$.

Si relacionamos el sexo con la dependencia funcional, se obtiene que las mujeres tienen una dependencia severa $35,5 \%$ (39) y los hombres una dependencia moderada con el $27,7 \%(28)$.

En cuanto a la correlación entre la edad de la población diana y el Barthel se obtiene que a mayor edad, mayor dependencia ( $r=-0,357 ; p=0,001)$. Las puntuaciones medias y desviación estándar del test de Barthel por grupos de población se especifican en la figura 6. La media de edad de la cuidadora familiar es $59 \pm 13$ años, de las cuales, 96 son mujeres y 19 hombres. El 40\% (46) de las cuidadoras familiares no tienen sobrecarga, el $38,3 \%$ (44) tienen sobrecarga intensa y el $21,7 \%$ (25) tiene sobrecarga leve.

Al comparar la dependencia del paciente (Barthel) con la sobrecarga del cuidador (Zarit) se obtiene una correlación de $(r=-201 ; p=0,031)$ es decir cuanta más dependencia de la persona, mayor sobrecarga para el cuidador familiar. 


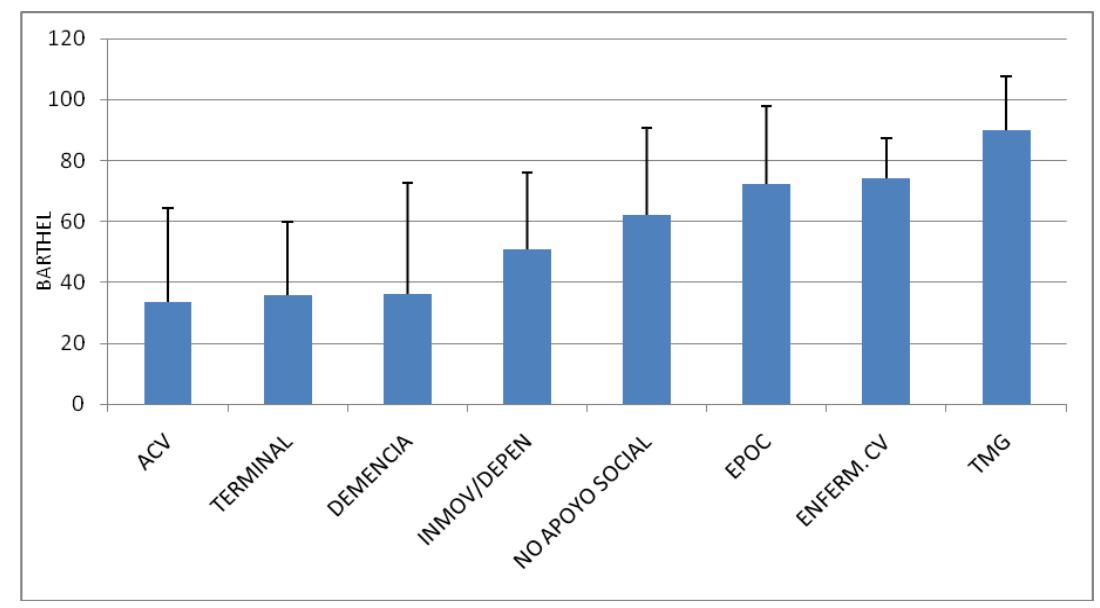

Figura 6. Fuente: elaboración propia. Gráfico de barras. Relación de medias y desviación estándar entre población diana y Barthel.

\section{DISCUSIÓN Y CONCLUSIONES}

Para una mejor comprensión, dividiremos esta sección según los objetivos planteados: Descripción de los procesos clave, Características sociodemográficas, capacidad funcional de la población diana y sobrecarga del cuidador.

Han sido captados 258 pacientes y 115 cuidadoras familiares. Supone un 5,3\% de la población compleja, es decir que se ha captado en un año toda la población compleja susceptible de gestión de casos del área IX. La mayoría de captaciones se han realizado desde AP, la población más frecuente ha sido EPOC seguido por ACV. Cumpliendo como criterios de inclusión 3 del $\mathrm{C}$ es decir, cumplir 3 de los cuatro posibles: oxigenoterapia domiciliaria, ser mayor de 65 años, anciano frágil, o estar polimedicado con más de 5 fármacos.

No se han derivado casos desde salud mental aunque la población con TMG ocupa el cuarto lugar, principalmente se han derivado desde la comunidad. La gestión de casos logra así un vínculo entre la comunidad y el sistema de atención sanitaria para este tipo de población, ya que la no adherencia al tratamiento de estas personas, entre otros factores, puede ocasionar que se desvinculen del sistema sanitario.

Las captaciones desde atención hospitalaria ocupan el segundo lugar y han derivado principalmente a la población sin cuidados familiares o apoyo social. El criterio de inclusión más frecuente es tener una deficiente red de apoyo social. Por ello estos resultados nos pueden orientar en que los profesionales, por la escasa tradición con este modelo de trabajo, ven la figura de la EGC como muy relacionada con la figura de la trabajadora social. Por otro lado, también podría interpretarse que ven necesaria la figura de la EGC para la coordinación con la comunidad una vez dado de alta. Sería necesario otro tipo de estudio para analizar la causa de estos resultados.

Hay que destacar que, desde la incorporación de la EGC, se ha mejorado el registro en $\mathrm{OMI}$ de las valoraciones por patrones, tanto de pacientes como de cuidadoras. En cuanto a la valoración por patrones funcionales, se ha obtenido que el patrón actividad y ejercicio, seguido de percepción y manejo de la salud, son los más alterados en la población diana. Un estudio realizado por enfermeras de enlace en 
Cataluña muestra que la necesidad de movimiento es la que está alterada más frecuentemente entre la población diana ${ }^{16}$.

Según el grupo de población, las personas con ACV presentan más patrones alterados, seguido de la población inmovilizada/dependiente. De los resultados obtenidos señalamos a la población con TMG que presenta como patrones alterados el cognitivo/perceptivo y rol/relaciones. Un estudio realizado con esta población, aunque llevado a cabo en salas de psiquiatría de hospitales generales, ofrece resultados similares ${ }^{17}$. Esta población con TMG, destaca porque se le ha realizado menos valoraciones completas, para ello señalar que los patrones funcionales que están en OMI no están adaptados para el grupo de población de salud mental por lo que la valoración se hace más complicada. Existe el modelo de Fornés $\mathrm{J}^{18}$ que sería más adecuada para este grupo de población.

Las cuidadoras familiares presentan como patrón más alterado el de actividad y ejercicio, seguido de percepción y manejo de la salud, al igual que ocurre en los pacientes. El que exista mayor frecuencia de registro de valoraciones y planes de cuidados se podría atribuir a que quizás por nuestra formación, existe mayor concienciación en el registro en la historia clínica y estamos más adiestradas para ello. También al ser sólo dos enfermeras, existe menos variabilidad en la recogida de datos y el registro de ellos.

Sí se podrían plantear otros estudios diseñados con el fin de observar la causa de la menor frecuencia y mayor variabilidad de registro en la historia clínica de OMI por parte de los enfermeros de AP. Con este estudio se muestra que el realizar una planificación de las acciones y registrarlas, implica la responsabilidad sobre las propias acciones y la necesidad de reconocerlas ${ }^{19}$.

La población de estudio tiene una dependencia severa para las ABVD. La población con demencia y ACV presenta dependencia total, y tienen una dependencia severa los inmovilizados, terminales y los que no tienen apoyo social, mientras que las personas con TMG son las más independientes para las ABVD. En general los resultados nos dicen que a mayor edad mayor dependencia. En otros estudios, la dependencia de la población diana de la EGC es una dependencia moderada ${ }^{16,20}$, mientras que la población de la EGC del SMS es más dependiente para las ABVD, esto puede deberse a que nuestra población está más envejecida.

Como conclusión de los datos obtenidos en este apartado, decir que la población diana derivada a la EGC es una población envejecida, frágil, con un nivel de dependencia severo para las ABVD, preferentemente es mujer, con cuidadora familiar (también mujer) donde son más frecuentes las que no tienen sobrecarga aunque las cuidadoras que tienen sobrecarga intensa también presentan un porcentaje elevado en este caso.

Este estudio nos ha mostrado una detección de problemas en poblaciones vulnerables como son las cuidadoras familiares. El cuidado de personas mayores dependientes se ha demostrado perjudicial para la salud de las personas cuidadoras $^{21}$. Es fundamental situarlas en una posición prioritaria de actuación creando líneas de trabajo conjuntas entre servicios de atención social y sanitaria. Para ello, la gestión de casos se presenta como una alternativa a tener en cuenta. 


\section{BIBLIOGRAFÍA}

(1) Rufete Gómez J. Curso Básico en Gestión de Casos. Enfermeras Gestoras de Casos. Murcia [Internet]. 2010 [consultado el 11 julio 2011]. disponible en: http://www.ffis.es/ups/enfermera\%20gestora\%20de\%20casos/COORDINACION\%20S OCIOSANITARIA.pdf

(2) Hunter DJ. Bridhing the Gap. Case management and Advocacy for People with Physical Handicaps. King's Fund Publishing office London; 1988: 105-110.

(3) Ewers M, Schaeffer D. Case Management in Theorie und Praxis. Bern, Göttingen, Toronto, Seattle; 2000

(4) Case Management Society of America (CMSA). Standars of Practise for Case Management. 2010.

(5) Sarabia Sánchez A. La gestión de casos como nueva forma de abordaje de la atención a la dependencia funcional. Revista Zerbituan, [revista en Internet]. 2007 Dic [consultado 22 Marzo 2011]; (42). Disponible en: http://www.zerbitzuan.net/documentos/zerbituan/La\%20gestion\%20\%20casos.pdf

(6) Bulechek GM, Butcher HK, McCloskey Dochterman J. Clasificación de Intervenciones de enfermeria (NIC).Barcelona: Elsevier; 2009

(7) Del Pino Casado R, Martínez Riera JR. Estrategias para mejorar la visibilidad y accesibilidad de los cuidados enfermeros en Atención Primaria de Salud. Rev. Adm Sanitar. 2007; 5(2):311-37.

(8) Ollero M. Modelos de organización para la atención a pacientes crónicos complejos. Foro de debate. Gestión clínica 2.0. Escuela Andaluza de Salud Pública.[Internet] 2009 [consultado el 17 agosto de 2011]; disponible en: http://si.easp.es/gestionclinica/wp-content/uploads/2009/10/Doc-2.-Modelos-de-

organizacion-para-la-atencion-a-pacientes-cronicos-complejos-_Ollero-Baturone-

M._pdf.

(9) Gala Fernández B, López Alonso SR, Pérez Hernández RM. La enfermera, profesional clave para la coordinación de la atención socio-sanitaria a personas con dependencia. Index de Enfermería [Index Enferm] (edición digital) 200654. [Consultado el 15 de 8 2011]Disponible en: http://www.index-f.com/indexenfermeria/54/0709.php.

(10) Plan para la mejora y modernización de la Atención Primaria 2007-2010. Murcia. 2007.

(11) Mora Martínez J.R, Ferrer Arrendó C, Ramos Quirós E. Gestión Clínica por procesos: mapa de procesos de enfermería en centros de salud. Administración Sanitaria. 2002; 21(6): 135-159.

(12) Servicio de Calidad de la Atención Sanitaria. Gestión por procesos. SESCAM.[Internet] 2002 [consultado 14 agosto 2011] disponible en: http://sescam.jccm.es/web1/profesionales/Calidad/GESTION_PROCESOS_SECAM.p df

(13) Dirección General de Asistencia Sanitaria. Enfermera Gestora de Casos en el Servicio Murciano de Salud. Programa Pilotaje [Internet] 2010 [consultado el 11 marzo 2011] disponible en: http://www.ffis.es/ups/taller_gestion_casos_2010/triptic_formacion_harvard_2009_4.p df

(14) Método epidemiológico. Manual Docente de la Escuela Nacional de Sanidad. Instituto Carlos III. Ministerio de Ciencia e Innovación. Madrid. 2009.

(15) Dirección General de Asistencia Sanitaria. Proyecto de Evaluación Pilotaje de enfermera gestora de casos en el Servicio Murciano de salud. 2010 
(16) Jurado-Campos J, Anglada Dilme MT, Canet Ponsa M, Privat Marcé M, Fábrega Pairó T, Juvinyá Canal D. Implementación de un modelo integrado de enfermería de enlace: un estudio descriptivo. Enferm Clín. 2008; 18(5):253-61.

(17) Sales R. Análisis del proceso de cuidados de enfermería en una sala de psiquiatría.[tesis doctoral]. Sevilla: Facultad de Medicina [internet][consultado 27 de septiembre de 2011] Disponible en: http://www.san.gva.es/val/prof/enfermeria/observatorio/tesistotal.pdf

(18) Fornes J, Carballal MC. Enfermería en salud mental y psiquiátrica. Guía para la valoración y estrategias de intervención. Madrid: Interamericana. 2001.

(19) Espinosa C. VII simposium internacional de diagnósticos de enfermería. Cuidar traspasando el diagnóstico enfermero. Barcelona. 2008.

(20) Mañes-López E, Benachi-Sandoval N, López-Poyato M, Martínez-Castellón E, Juan-Andrés C. Estudio descriptivo de la tipología de pacientes atendidos en un programa de enfermeras de enlace. Enferm Clín. 2009; 19(6):306-313.

(21) Del Pino Casado R, Frías Osuna A, Palomino Moral PA, Millán Cobo MD. Diferencias de género en la sobrecarga e implicación en el cuidado familiar de mayores dependientes. Revista Iberoamericana de Enfermería Comunitaria. 2010; $3(2): 76-82$. 